\title{
Similarities between extreme events in the solar-terrestrial system by means of nonextensivity
}

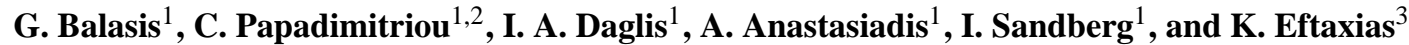 \\ ${ }^{1}$ Institute for Space Applications and Remote Sensing, National Observatory of Athens, Greece \\ ${ }^{2}$ Section of Astrophysics, Astronomy and Mechanics, Department of Physics, University of Athens, Greece \\ ${ }^{3}$ Section of Solid State Physics, Department of Physics, University of Athens, Greece
}

Received: 31 January 2011 - Revised: 27 May 2011 - Accepted: 22 August 2011 - Published: 5 September 2011

\begin{abstract}
The dynamics of complex systems are founded on universal principles that can be used to describe disparate problems ranging from particle physics to economies of societies. A corollary is that transferring ideas and results from investigators in hitherto disparate areas will cross-fertilize and lead to important new results. In this contribution, we investigate the existence of a universal behavior, if any, in solar flares, magnetic storms, earthquakes and pre-seismic electromagnetic (EM) emissions, extending the work recently published by Balasis et al. (2011a). A common characteristic in the dynamics of the above-mentioned phenomena is that their energy release is basically fragmentary, i.e. the associated events are being composed of elementary building blocks. By analogy with earthquakes, the magnitude of the magnetic storms, solar flares and pre-seismic EM emissions can be appropriately defined. Then the key question we can ask in the frame of complexity is whether the magnitude distribution of earthquakes, magnetic storms, solar flares and pre-fracture EM emissions obeys the same law. We show that these apparently different extreme events, which occur in the solar-terrestrial system, follow the same energy distribution function. The latter was originally derived for earthquake dynamics in the framework of nonextensive Tsallis statistics.
\end{abstract}

\section{Introduction}

A central property of the magnetic storm, solar flare, and earthquake preparation process is the possible occurrence of coherent large-scale collective behavior with a very rich structure resulting from the repeated nonlinear interactions among their constituents taking place in the magnetosphere (Chang et al., 2003, 2006, 2010; Consolini et al., 2005;

Correspondence to: G. Balasis

(gbalasis@space.noa.gr)
Wanliss, 2005, Wanliss et al., 2005; Balasis et al., 2006), solar corona (Vassiliadis et al., 1998; Isliker et al., 2001; Baiesi et al., 2006) and lithosphere (Turcotte, 1997; Sornette, 2004; Eftaxias et al., 2009, 2010), respectively. Nonextensive statistical mechanics provides a solid theoretical basis for describing and analyzing complex systems out of equilibrium, systems exhibiting long-range correlations, memory, or fractal properties (Tsallis, 2009). Recently, there have been promising applications of the nonextensive Tsallis statistics (Tsallis, 1988, 2009) to magnetic storms (Balasis et al., 2008, 2009; Balasis and Eftaxias, 2009), solar wind (Leubner and Vörös, 2005), solar flares (Balasis et al., 2011a), crustal seismicity (Telesca, 2010; Telesca and Chen, 2010) and preseismic EM emissions (Kalimeri et al., 2008; Papadimitriou et al., 2008). Consequently, the nonextensive statistical mechanics is an appropriate mathematical tool to investigate universality, if any, associated with the occurrence of magnetic storms, solar flares, earthquakes and pre-failure EM emissions in the solar-terrestrial system.

A model for earthquake dynamics consisting of two rough profiles interacting via fragments filling the gap has been recently introduced by Sotolongo-Costa and Posadas (2004). Based on this model, an energy distribution function, which gives the Guttenberg-Richter empirical law (Guttenberg and Richter, 1944) as a particular case, has been analytically deduced in the framework of Tsallis statistical mechanics (Sotolongo-Costa and Posadas, 2004). More recently, Silva et al. (2006) have revised the model introduced by Sotolongo-Costa and Posadas (2004). Their analysis resulted in a different nonextensive Guttenberg-Richter type law. The proposed Guttenberg-Richter type laws in Sotolongo-Costa and Posadas (2004) and Silva et al. (2006) provide an excellent fit to seismicities generated in various large geographic areas usually identified as "seismic regions", each of them covering many geological faults. We emphasize that the empirical statistical relationship by Guttenberg-Richter does not bury any information about a specific activated fault. 
The primary question we can ask in the context of complex systems theory is whether the aforementioned nonextensive laws not only successfully describe the magnitude distribution of earthquakes in various seismic regions in Earth but also magnetic storms, solar flares and pre-seismic EM emissions, rooted in the activation of a single fault, in the solar-terrestrial system. A subsequent question is whether these laws successfully describe the magnitude distribution in all the cases under study with a similar nonextensive entropic parameter $q$. We show that both key questions accept a positive answer. It is worth mentioning that the estimated $q$ nonextensive parameter values are in full agreement with the upper limit, $q<2$, obtained from several independent studies involving the Tsallis nonextensive framework (Tsallis, 2009).

\section{Principles of Tsallis statistical mechanics}

The aim of statistical mechanics is to establish a direct link between the mechanical laws and classical thermodynamics. One of the crucial properties of the Boltzmann-Gibbs entropy in the context of classical thermodynamics is extensivity, namely proportionality with the number of elements of the system. The Boltzmann-Gibbs entropy satisfies this prescription if the subsystems are statistically (quasi-) independent, or typically if the correlations within the system are essentially local. In such cases the system is called extensive. In general, however, the situation is not of this type and correlations may be far from negligible at all scales.

It has been established that physical systems, which are characterized by long-range interactions or long-term memories, or are of a multi-fractal nature, are best described by a generalized statistical-mechanical formalism proposed by Tsallis (1988, 2009). More precisely, inspired by multifractals concepts, Tsallis introduced an entropic expression characterized by an index $q$, which leads to nonextensive statistics $(1988,2009)$ :

$S_{q}=k \frac{1}{q-1}\left(1-\sum_{i=1}^{W} p_{i}^{q}\right)$

where $p_{i}$ are probabilities associated with the microscopic configurations, $W$ is their total number, $q$ is a real number called the entropic index and $k$ is Boltzmann's constant.

The entropic index describes the deviation of Tsallis entropy from the standard Boltzmann-Gibbs entropy. Indeed, using $p_{i}^{(q-1)}=e^{(q-1) \ln \left(p_{i}\right)} \sim 1+(q-1) \ln \left(p_{i}\right)$ in the limit $q \rightarrow 1$, we recover the usual Boltzmann-Gibbs entropy:

$S_{1}=-k \sum_{i=1}^{W} p_{i} \ln \left(p_{i}\right)$.

The entropic index $q$ characterizes the degree of nonextensivity reflected in the following pseudo-additivity rule:

$S_{q}(A+B)=S_{q}(A)+S_{q}(B)+\frac{1-q}{k} S_{q}(A) S_{q}(B)$.
For subsystems that have special probability correlations, extensivity

$S_{1}(A+B)=S_{1}(A)+S_{1}(B)$

is not valid for $S_{1}$, but may occur for $S_{q}$ with a particular value of the index $q$. Such systems are sometimes referred to as nonextensive (Tsallis, 1988, 2009).

The cases $q>1$ and $q<1$, correspond to sub-additivity, or super-additivity, respectively. We may think of $q$ as a biasparameter: $q<1$ privileges rare events, while $q>1$ privileges prominent events.

\section{Calculation of Tsallis entropy using symbolic dynamics methods}

The basic idea of symbolic dynamics is simple. One divides the phase space into a finite number of partitions and labels each partition with a symbol (e.g. a letter from some alphabet). Instead of representing the trajectories by infinite sequences of numbers-iterates from a discrete map or sampled points along the trajectories of a continuous flow, one watches the alteration of symbols. Of course, in so doing one loses an amount of detailed information, but some of the invariant, robust properties of the dynamics may be kept, e.g. periodicity, symmetry, or the chaotic nature of an orbit (Hao, 1989).

In the framework of symbolic dynamics, time series are transformed into a series of symbols by using an appropriate partition, which results in relatively few symbols. After symbolization, the next step is the construction of "symbol sequences" ("words" in the language symbolic dynamics) from the symbol series by collecting groups of symbols together in temporal order.

Herein, we estimate $S_{q}$ based on the concept of symbolic dynamics: from the initial measurements we generate a sequence of symbols, where the dynamics of the original (under analysis) system has been projected (Hao, 1989). More precisely, the original $D_{\text {st }}$ time series of length $N,\left(X_{1}, X_{2}, \ldots, X_{N}\right)$, is projected to a symbolic time series $\left(A_{1}, A_{2}, \ldots, A_{N}\right)$ with $A_{n}$ from a finite alphabet of $\lambda$ letters $(0, \ldots, \lambda-1)$ (see for example Balasis et al., 2008, 2009).

After symbolization, the next step in identification of temporal patterns is the construction of symbol sequences with size $L$. We use the technique of lumping. Thus, we stipulate that the symbolic sequence is to be read in terms of distinct successive "blocks" of length $L$, $A_{1}, A_{2}, \ldots, A_{L} / A_{L+1}, \ldots, A_{2 L} / A_{j L+1}, \ldots, A_{(j+1) L}$.

The number of all possible blocks of length $L$ in a $\lambda$-letter alphabet is $N_{\lambda}=\lambda^{L}$. We determine the probabilities of occurrence of each of $N_{\lambda}$ different kind of blocks,

$p(L)_{A_{1}, A_{2}, \ldots, A_{L}}=$

Number of blocks of the form $A_{1}, A_{2}, \ldots, A_{L}$ encounter by lumping Total number of blocks encountered by lumping

To be more concrete, the simplest possible coarse graining of the $D_{\text {st }}$ index is given by choosing a threshold $C$ 
(usually the mean value of the data considered) and assigning the symbols " 1 " and " 0 " to the signal, depending on whether it is above or below the threshold (binary partition). Thus, we generate a symbolic time series from a 2-letter $(\lambda=2)$ alphabet $(0,1)$, e.g. $0110100110010110 \ldots$ Reading the sequence by lumping of length $L=2$ one obtains $01 / 10 / 10 / 01 / 10 / 01 / 01 / 10 / \ldots$. The number of all possible kinds of blocks is $\lambda^{L}=2^{2}=4$, namely $00,01,10,11$. Thus, the required probabilities for the estimation of the Tsallis entropy $p_{00}, p_{01}, p_{10}, p_{11}$ are the fractions of the blocks 00 , $01,10,11$ in the symbolic time series.

The $S_{q}$ for the word length $L$ is

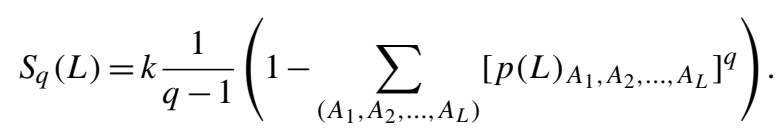

Broad symbol-sequence frequency distributions produce high entropy values, indicating a low degree of organization. Conversely, when certain sequences exhibit high frequencies, lower entropy values are produced, indicating a high degree of organization.

\section{A nonextensive model for earthquake dynamics}

The nonextensive formulation (1) seems to present an appropriate theoretical tool to investigate complex systems in their nonequilibrium stationary states, systems with multifractal and self-similar structures, systems dominated by long-range interactions, as well as anomalous phenomena.

The process of shock fragmentation associated to earthquakes, especially when energies are high enough, leads to the existence of long-range correlations between all parts of the object being fragmented. Then the use of a nonextensive approach seems to be appropriate.

Guttenberg and Richter (1944) proposed an empirical law that expresses the relationship between the magnitude and total number of earthquakes in any given region and time period of at least that magnitude

$\log (N(>m))=a-b m$,

where $N$ is the number of events having a magnitude greater than $m$ and $a$ and $b$ are constants.

Now, we focus on the seismicity model proposed by Sotolongo-Costa and Posadas (2004) in the framework of Tsallis statistical mechanics. Its theoretical ingredients read as follows:

1. The mechanism of relative displacement of fault plates is the main cause of earthquakes.

2. The space between fault planes is filled with the residues of the breakage of the tectonic plates, from where the faults have originated.
3. The motion of the fault planes can be hindered not only by the overlapping of two irregularities of the profiles, but also by the eventual relative position of several fragments. Thus, the mechanism of triggering earthquakes is established through the combination of the irregularities of the fault planes on one hand and the fragments between them on the other hand.

4. The fragments-distribution function, and consequently the energy-distribution function, emerges naturally from a nonextensive framework starting from first principles, i.e. the maximum entropy formalism.

In the context of Sotolongo-Costa and Posadas model (2004) Tsallis entropy has the form

$S_{q}=k \frac{1-\int p^{q}(\sigma) d \sigma}{q-1}$,

where $p(\sigma)$ stands for the probability of finding a fragment of relative surface $\sigma$ (which is defined as a characteristic surface of the system in Sotolongo-Costa and Posadas, 2004). The maximum entropy formulation for Tsallis entropy involves the introduction of the following two constraints. The first one is the normalization of $p(\sigma)$ :

$\int_{0}^{\infty} p(\sigma) d \sigma=1$

and the other is the ad hoc condition about the $q$-mean value, which can be expressed as

$\int_{0}^{\infty} \sigma p^{q}(\sigma) d \sigma=<<\sigma>_{q}$.

Finally, based on the extremization of the entropy functional, Sotolongo-Costa and Posadas (2004) obtained the following analytic expression for the energy distribution of earthquakes:

$$
\begin{aligned}
& \log (N(>m))=\log N+\left(\frac{2-q}{1-q}\right) \times \\
& \log \left[1+\alpha(q-1) \times(2-q)^{(1-q) /(q-2)} 10^{2 m}\right],
\end{aligned}
$$

where $N$ is the total number of earthquakes, $N(>m)$ the number of earthquakes with magnitude larger than $m$, and $m \approx \log (\varepsilon)$. This is not a trivial result, and incorporates the characteristics of nonextensivity into the distribution of earthquakes by magnitude. $\alpha$ is the constant of proportionality between the earthquake energy, $\varepsilon$, and the size of fragment, $r$. More precisely, Sotolongo-Costa and Posadas (2004) assume that $\varepsilon \propto r$.

Sotolongo-Costa and Posadas (2004) successfully used Eq. (9) to describe the relative cumulative number of earthquakes to different seismic regions with $q$ values ranging from 1.6 to 1.65 . It is very important to observe the similarity in the value of the nonextensivity parameter $q$ for the three seismic catalogs of the different seismic regions 

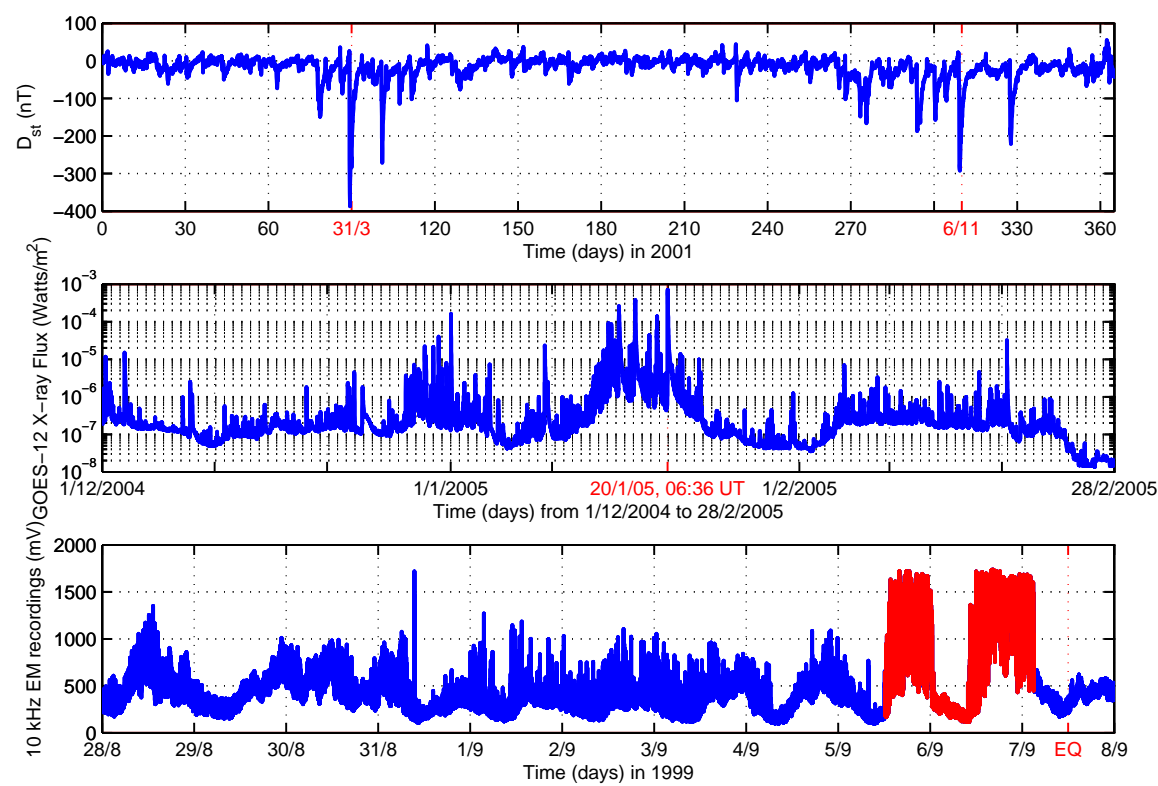

Fig. 1. $D_{\text {st }}$ time series (upper panel). The 31 March and 6 November 2001 magnetic storms are marked with red. GOES-12 5-min averages X-ray flux, $X_{l}$ (1-8 Angstrom) time series (middle panel). The 20 January 2005 solar flare is marked with red. Electromagnetic time series recorded at $10 \mathrm{kHz}$ (lower panel). The pre-seismic EM emissions are shown in red. The 7 September 1999 Athens earthquake is marked with red.

used. We note that Eq. (9) describes the energy distribution in the full range of detectable (earthquake) magnitudes very well. On the contrary, for the smallest and largest magnitudes the empirical formula of Guttenberg-Richter fails to describe the seismic data (Kossobokov et al., 2000; Sornette and Helmstetter, 2002).

As it is mentioned, Silva et al. (2006) have revised the fragment-asperity interaction model introduced by Sotolongo-Costa and Posadas (2004). They consider the current definition of the mean value, i.e. the so-called $q$ expectation value (see Abe and Bagci, 2005 for details). Moreover, they introduce a different scale between the size of the released relative energy $\varepsilon$ and the size of fragment $r$, i.e. $\varepsilon \propto r^{2}$. The magnitude-distribution function deduced in their approach is given by

$$
\begin{aligned}
\log (N(>m))= & \log N+\left(\frac{(2-q)}{1-q}\right) \times \\
& \log \left[1-\left(\frac{1-q}{2-q}\right)\left(\frac{10^{2 m}}{\alpha^{2 / 3}}\right)\right],
\end{aligned}
$$

which is different from Eq. (9) obtained by Sotolongo-Costa and Posadas (2004).

Silva et al. (2006) successfully tested the viability of this distribution function with data in various areas with $q$ varying between 1.6 and 1.7. By using the Tsallis-based nonextensive statistics, the analysis of the magnitude distribution of several seismic catalogues in Italy was performed (Telesca,
2010). Those results could provide hints for further investigation in discriminating tectonic from volcanic seismicity. Furthermore, Telesca and Chen (2010) found that the nonextensive statistics furnishes a very good prediction of the cumulative magnitude distribution of crustal seismicity in Taiwan. Matcharashvili et al. (2011) investigated the seismic catalogues of Southern Caucasus using a nonextensive statistical approach and found a $q$ parameter equal to 1.81 , a quite important value also from our point of view as it is shown next.

\section{Application of the earthquake nonextensive model to magnetic storms, solar flares and pre-seismic EM emissions}

Magnetic storms are initiated when enhanced energy transfer from the solar wind (and the associated interplanetary magnetic field-IMF) into the magnetosphere leads into intensification of the ring current that flows around the Earth (e.g. Daglis, 2006). Magnetic storm intensity is usually represented by an average of the geomagnetic perturbations measured at four mid-latitude magnetic observatories, known as the hourly $D_{\text {st }}$ index (http://swdcwww.kugi.kyoto-u.ac.jp/), which serves as a proxy of the ring current intensity. In this study, we consider 1-yr $D_{\text {st }}$ data (2001) that include two intense magnetic storms, which occurred on 31 March 2001 (when $D_{\text {st }}$ reached $-387 \mathrm{nT}$ ) and 6 November 2001 (with a $D_{\text {st }}$ of $-292 \mathrm{nT}$ ) (Fig. 1, upper panel). 

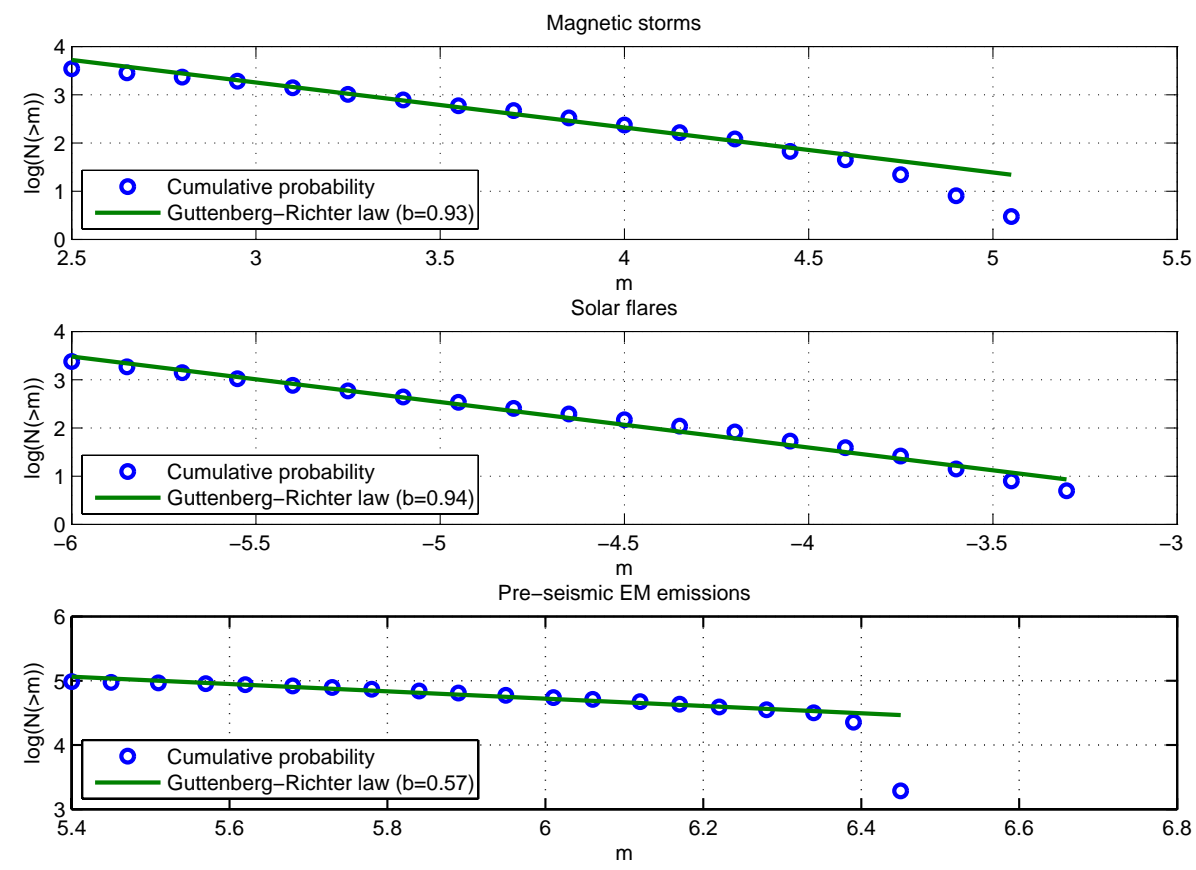

Fig. 2. We use the Gutenberg-Richter law (Eq. 5) to calculate the cumulative number of $D_{\text {st }}$ data, $N(>m$ ) (upper panel). There is an agreement of the aforementioned formula for $b=0.93$ with the $D_{\text {st }}$ time series. We then use the Gutenberg-Richter law to calculate the cumulative number of X-ray flux data, $N(>m)$ (middle panel). There is an agreement of the aforementioned formula for $b=0.94$ with the X-ray flux time series. Finally, the Gutenberg-Richter law is used to calculate the cumulative number of pre-seismic EM emission data, $N(>m)$ (lower panel). There is an agreement of the aforementioned formula for $b=0.57$ with the pre-seismic EM emission time series.

Solar flares are highly energetic explosions from active regions of the Sun in the form of EM radiation, energetic particle and plasma flows powered by strong and twisted magnetic fields. A solar flare occurs when magnetic energy that has built up in the solar atmosphere is suddenly released. In particular, radiation is emitted across virtually the entire EM spectrum, from radio waves at the long-wavelength end, through optical emission to X-rays and $\gamma$-rays at the shortwavelength end. Solar flares are classified as A, B, C, M or $\mathrm{X}$ according to the peak flux (in $\mathrm{W} \mathrm{m}^{-2}$ ) of $100-800 \mathrm{pm} \mathrm{X-}$ rays near the Earth, as measured on the GOES spacecraft. Each class has a peak flux ten times greater than the preceding one, with X-class flares having a peak flux of the order of $10^{-4} \mathrm{~W} \mathrm{~m}^{-2}$. The more powerful $\mathrm{M}$ - and X-class flares are often associated with a variety of effects on the near-Earth space environment. Herein, we consider 3 months of GOES$12 \mathrm{X}$-ray fluxes (5-min averages detected in 1-8 Angstrom) (Fig. 1, middle panel). We focus on a series of M- and Xclass solar flares, which occurred in the single extensive active region 0720 between 10 and 23 January 2005. In particular, the solar flare that occurred on 20 January 2005 released the highest concentration of protons ever directly measured.

Earthquakes are large-scale fracture phenomena in the Earth's heterogeneous crust. Herein, we focus on the case of Athens earthquake $\left(M_{\mathrm{S}}=5.9\right)$ that occurred on 7 September 1999. EM anomalies at $10 \mathrm{kHz}$ were detected from a few days up to a few hours prior to this earthquake (Kapiris et al., 2004a). The seismogenic origin of this EM activity has been supported by a series of papers (Eftaxias et al., 2001, 2004, 2007; Kapiris et al., 2004a, b, 2005; Karamanos et al., 2005, 2006; Contoyiannis et al., 2005). The candidate precursory $10 \mathrm{kHz}$ EM emission emerged on 1 September 1999 (Karamanos et al., 2005, 2006) (Fig. 1, lower panel, signal in red). Figure 1 shows that the precursor launches from a long duration quiescence period concerning the detection of EM disturbances at the $\mathrm{kHz}$ frequency band (Fig. 1, lower panel, all data). This category of EM anomalies are related to the coupled Earth's ionosphere, atmosphere and lithosphere system (Pulinets and Boyarchuk, 2004).

Figure 2 explores the applicability of the GuttenbergRichter law (Eq. 5) to the $D_{\text {st }}$, X-ray flux and kHz EM anomaly data shown in Fig. 1, i.e. to the energy associated to magnetic storms, solar flares and pre-seismic EM emissions, respectively. In the case of X-ray flux we take the integral of fluxes for calculating energy, whereas in the case of $D_{\mathrm{st}}$ the square of the amplitude of the index (i.e. the square of the geomagnetic field) is proportional to energy. Regarding the $\mathrm{kHz}$ EM anomalies we only consider the red part of the signal (cf. Fig. 1 lower panel), which is transformed into energy by taking the square of the voltage. At each panel of Fig. 2, $N$ is the total number of data and $N(>m)$ the number of values exceeding $m$. The threshold is $-30 \mathrm{nT}$ for the $D_{\mathrm{st}}$ index, which 

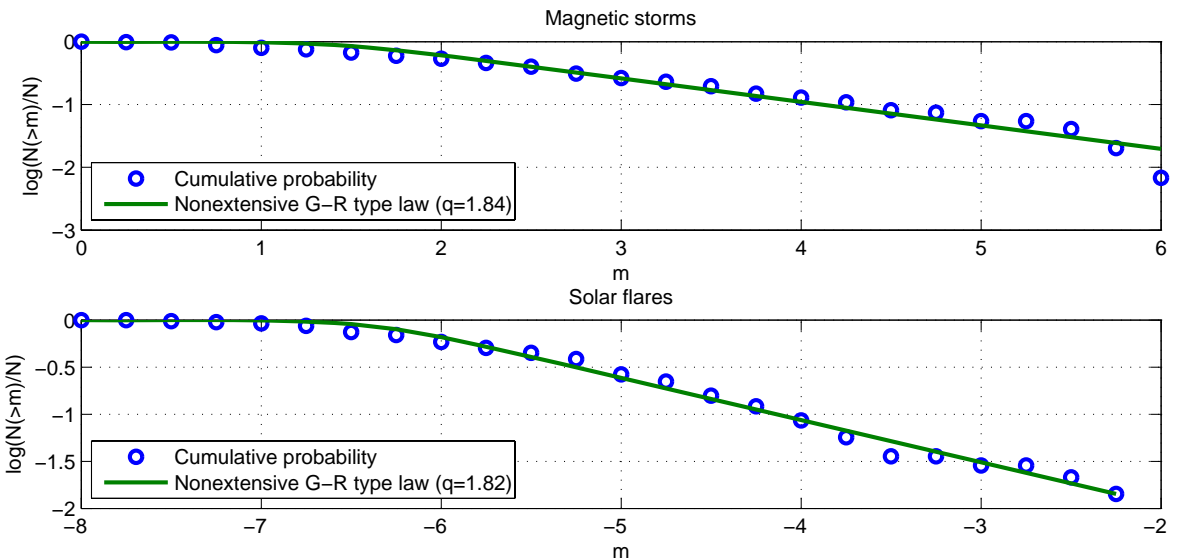

Pre-seismic EM emissions

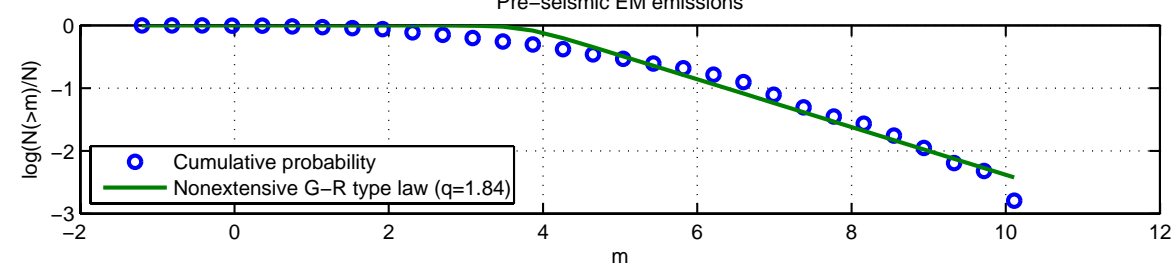

Fig. 3. We use the Gutenberg-Richter (G-R) type law for the nonextensive Tsallis statistics (Eq. 10) to calculate the relative cumulative number of $D_{\text {st }}$ data, $N(>m) / N$ (upper panel). There is an excellent agreement of the aforementioned formula with the $D_{\text {st }}$ time series. The threshold is $-30 \mathrm{nT}$, which results in 164 events, and the associated parameter is $q=1.84$. We then use Eq. (10) to calculate the relative cumulative number of X-ray flux data, $N(>m) / N$ (middle panel). There is an excellent agreement of the aforementioned formula with the $\mathrm{X}$-ray flux time series. The threshold is $10^{-6} \mathrm{~W} \mathrm{~m}^{-2}$, which results in 141 events, and the associated parameter is $q=1.82$. Finally, Eq. (10) is used to calculate the relative cumulative number of pre-seismic EM emission data, $N(>m) / N$ (lower panel). There is an excellent agreement of the aforementioned formula with the pre-seismic EM emission time series. The threshold is $500 \mathrm{mV}$, which results in 626 events, and the associated parameter is $q=1.84$.

results in 164 events, $10^{-6} \mathrm{~W} \mathrm{~m}^{-2}$ for the solar fluxes, which results in 141 events and $500 \mathrm{mV}$ for the $\mathrm{kHz}$ EM anomalies, which results in 626 events. For the case of the $D_{\text {st }}$ index, the energy associated with each data point is given by the square of the difference between the field value and a background noise level (threshold). For the case of X-ray data, where the measured quantity is the energy flux, there is no need to use the square of the data. In both cases, we consider a sequence of $N$ consecutive values that surpass the threshold to constitute an "event" and measure its energy by summing (integrating) the individual data-point energies that comprise it. We then use Gutenberg-Richter law to calculate the cumulative number of each kind of energies. There is an agreement of the Guttenberg-Richter formula for $b=0.93$ with the $D_{\text {st }}$ data, for $b=0.94$ with the X-ray fluxes and for $b=0.57$ with the pre-seismic EM emission time series.

Figure 3 explores the applicability of the nonextensive Guttenberg-Richter type law (Eq. 10) to the three different kinds of data used in Fig. 2, i.e. to the energy related to magnetic storms, solar flares and pre-seismic EM emissions, respectively. The various data are transformed to energies as in Fig. 2. For each category of extreme events, $N$ is the total number of data, $N(>m)$ the number of values exceeding $m$, $N(>m) / N$ the relative cumulative number of events with magnitude larger than $m$, and $\alpha$ a proportionality constant. There is an excellent agreement of the aforementioned formula with the $D_{\text {st }}$ time series (upper panel). The threshold is $-30 \mathrm{nT}$, which results in 164 events, and the associated Tsallis value is $q=1.84$. There is an excellent agreement of the aforementioned formula with the X-ray flux time series (middle panel). The threshold is $10^{-6} \mathrm{~W} \mathrm{~m}^{-2}$, which results in 141 events, and the associated Tsallis value is $q=1.82$. Finally, there is an excellent agreement of the aforementioned formula with the pre-seismic EM emission time series (lower panel). The threshold is $500 \mathrm{mV}$, which results in 626 events, and the associated Tsallis value is $q=1.84$.

We clarify that the parameter $q$ itself is not a measure of the complexity of the system but measures the degree of nonextensivity of the system. It is the time variations of the Tsallis entropy for a given $q\left(S_{q}\right)$ that quantify the dynamic changes of the complexity of the system. Lower $S_{q}$ values characterize the portions of the signal with lower complexity. Tsallis entropy, $S_{q}$, depends upon the choice of entropic index $q$ (Eq. 1). The application of the nonextensive Guttenberg-Richter type law (Eq. 10) to the data of Fig. 1 presents a way to derive appropriate $q$ values for calculating the corresponding Tsallis entropies for each phenomenon (Kalimeri et al., 2008; Balasis and Eftaxias, 2009). Figure 4 

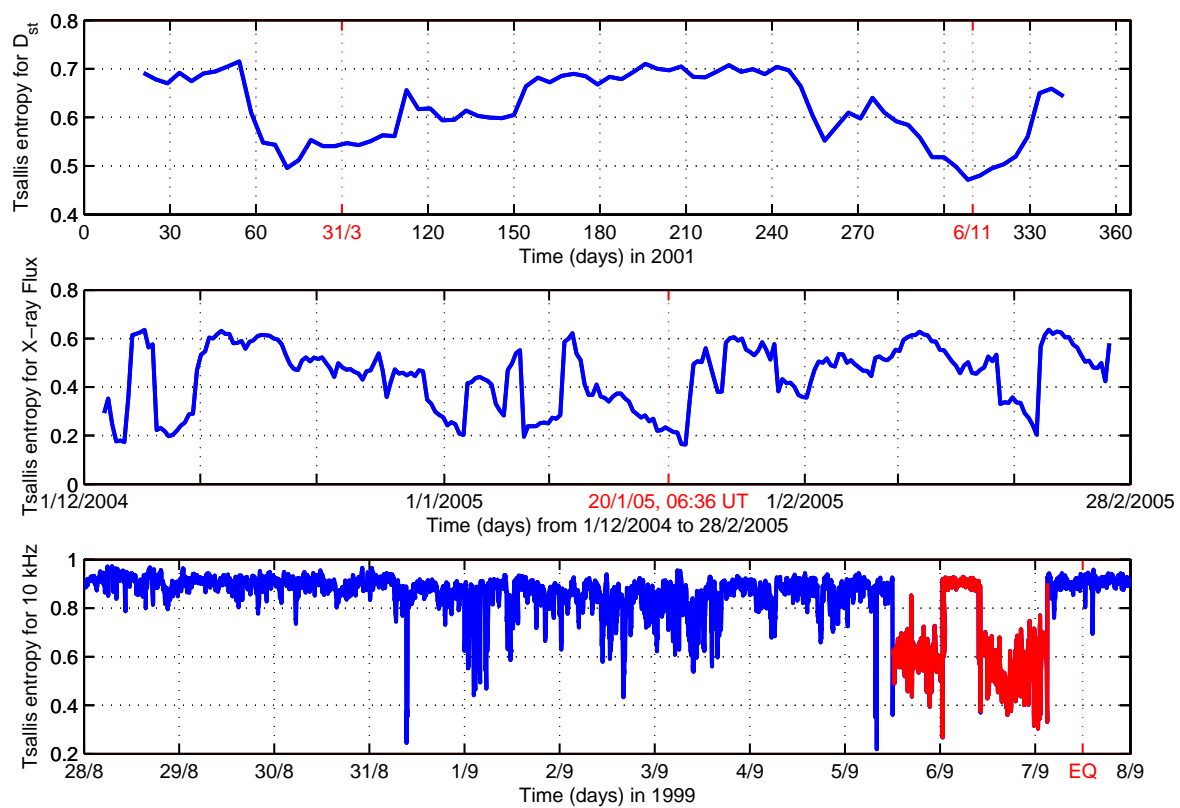

Fig. 4. Tsallis entropy variations of the $D_{\mathrm{st}}$ index data for $q=1.84$ (upper panel); X-ray flux data for $q=1.82$ (middle panel); $\mathrm{kHz}$ EM recordings data for $q=1.84$ (lower panel). The data correspond to the time series presented in Fig. 1. The red marks coincide with those of Fig. 1.

shows the temporal evolution of Tsallis entropy for the time series of $D_{\text {st }}$, X-ray flux and $\mathrm{kHz}$ EM recordings of Fig. 1. The calculation of the entropy values is based on the use of Tsallis parameters $q$ found when applying Eq. (10) to each type of data, i.e. 1.84 in the case of time series associated to magnetic storms, 1.82 in the case of time series associated to solar flares and 1.84 in the case of time series associated to pre-seismic EM emissions. We clarify that the computation of Tsallis entropy assumes stationarity for the signal. In practice, the condition of stationarity for non-stationary signals can be satisfied by dividing the signal into blocks of short, pseudo-stationary segments (Akay, 1997). Then Tsallis entropy values are calculated using formula (4) for the corresponding Tsallis parameter values.

Figure 4 shows that in time intervals around extreme events (i.e. intense magnetic storms of 31 March 2001 and 6 November 2001, solar flare of 20 January 2005, and earthquake of 7 September 1999) the corresponding Tsallis entropies of $D_{\text {st }}$ data, X-ray fluxes and EM emissions, respectively, attain lower values than for the rest of the time series. Lower entropy means lower complexity or a higher degree of organization for the corresponding natural system (magnetosphere, solar corona and coupled Earth's ionosphere, atmosphere and lithosphere system, respectively) around the particular extreme event.

One can observe in Fig. 4 (X-ray flux data) that Tsallis entropy is reaching low values $(0.2)$ a few times during the considered interval, but only one minimum is associated with a large flare. A possible explanation for this observation is related to the fact that a necessary condition for the occurrence of a large solar flare is the growth of the correlation length in the system. The occurrence of less intense solar flare events (as evidenced here by the decreases in Tsallis entropy) represents a footprint related to the increase of the correlation length in the system.

The three time series analyzed herein include both quiet and disturbed times of the magnetosphere, solar corona and coupled Earth's ionosphere, atmosphere and lithosphere system, respectively. The Tsallis entropy sensitively shows the complexity dissimilarity among different "physiological" (normal) and "pathological" states (intense magnetic storm, large solar flare and destructive earthquake). The Tsallis entropy implies the emergence of two distinct patterns at each time series: (i) a pattern associated with the above mentioned extreme events, which is characterized by a higher degree of organization, and (ii) a pattern associated with normal periods, which is characterized by a lower degree of organization.

\section{Conclusions}

Empirical evidence has been mounting that supports the possibility that a number of systems arising in disciplines as diverse as physics, biology, engineering, and economics may have certain quantitative features that are intriguingly similar. Universality relates to the uncovering of a universal formula that describes with good approximation the same property on different systems (Zhou et al., 2006). For instance, de Arcangelis et al. (2006) showed that the same empirical 


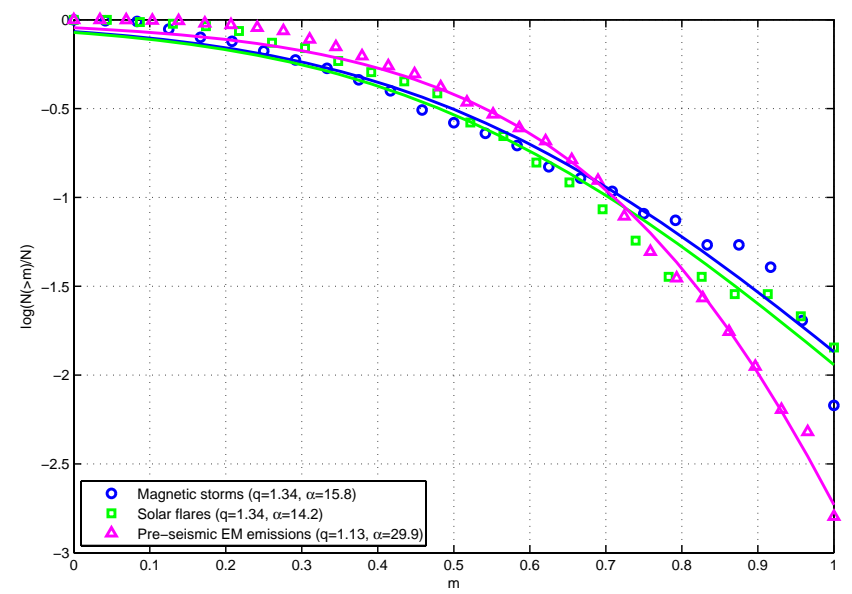

Fig. 5. Searching for the existence of a master universal curve by appropriately scaling the three different cumulative distribution functions of Fig. 4.

laws widely accepted in seismology also characterize, surprisingly, the size and time occurrence of solar flares. Recently, Balasis et al. (2011a) provided evidence for universality in solar flare, magnetic storm and earthquake dynamics. Herein, we extend that work to also include pre-seismic EM emissions.

First, the applicability of the Guttenberg-Richter law to the $D_{\text {st }}$, X-ray flux and kHz EM anomaly data is demonstrated. Then, we concentrate on signatures of universality in the solar-terrestrial system using the nonextensive Tsallis statistics (Tsallis, 1998). A previously derived equation describing earthquake dynamics of a single fault is used (Silva et al., 2006). This formula stands for a nonextensive GuttenbergRichter type law since it was developed in the framework of nonextensive Tsallis statistical mechanics. Moreover, direct Tsallis entropy variations are provided for corresponding time series of various categories of extreme events. We show that geomagnetic $D_{\text {st }}$ index time series around intense magnetic storms, X-ray flux data around large solar flares and $\mathrm{kHz} \mathrm{EM}$ emissions around strong earthquakes obey the same nonextensive energy distribution function. We thus provide evidence for universal behavior in the Earth's magnetosphere, solar corona and in the coupled Earth's ionosphere, atmosphere and lithosphere system where magnetic storms, solar flares and pre-seismic kHz EM anomalies occur, respectively. The presence of universality is further supported by the observation that all the different types of data considered here for the various phenomena obey the nonextensive Guttenberg-Richter type law with an almost identical Tsallis entropic parameter value $q$, which is equal to 1.8 .

Because the existence of a scaling ansatz would be interesting for its physical implications, we also search for the existence of a master universal curve by appropriately scaling the three different cumulative distribution functions of Fig. 4. The results are presented in Fig. 5. Magnetic storms and solar flares parameters used for fitting Eq. (10) to the data have quite close values while pre-seismic EM emissions parameters values are a bit different. There has been a series of attempts to search for common features in different natural phenomena. Kossobokov et al. (2000) explored the similarities of multiple fracturing on a neutron star and on the Earth. Starquakes provided the drastic extension of the realm of multiple fracturing previously observed in an already broad variety of conditions, from the lithosphere of the Earth through geotechnical and engineering constructions to laboratory samples of solid materials. Kossobokov et al. (2000) found similarities that may reflect a scenario of a critical transition, common for a broader class of nonlinear systems. Sornette and Helmstetter (2004) presented evidence of critical stochastic finite-time singularities in the rupture of heterogeneous materials, in the largest sequence of starquakes ever attributed to a neutron star, as well as in earthquake sequences. Another interesting observation that witnesses universality among different systems is the emergence of discrete scale invariance. Discrete scale invariance has been documented so far in ruptures, seismicity, financial systems (for a review see Sornette, 2004) and also quite recently for $D_{\text {st }}$ time series (Balasis et al., 2011b).

A number of authors (e.g. Nauenberg, 2003; Zanette and Montemurro, 2004; Lavenda and Dunning-Davies, 2003a, b) expressed different views on the significance of Tsallis entropy. However, since its launch more than 2 decades ago the theory of Tsallis statistics has been succesfully applied to a wide range of phenomena in diverse disciplines such as physics, chemistry, biology, medicine, economics, geophysics etc. Herein, we have applied Tsallis statistical mechanics in a variety of diverse natural phenomena in the solar-terrestrial system. The theory gives promising results with respect to a commonly observed behavior around extreme events related to these phenomena.

Efforts to infer a possible explanation for the observed common behavior of these extreme events in the solarterrestrial system poses a question about the origin of the common features observed in such different phenomena. The presented similarities suggest that these apparently diverse phenomena may follow qualitatively similar physical mechanisms. For instance, de Arcangelis et al. (2006) suggested that magnetic stress transfer in the solar corona plays the role of elastic stress redistribution on the Earths crust. We suggest that plasma pressure distribution in the inner magnetosphere could play the role of magnetic stress transfer in the solar corona and elastic stress redistribution on the Earths crust. Therefore, diagnostic and forecasting ideas and methodologies can be transferred from one discipline to another thus helping to improve the present status of knowledge at each discipline. 
Acknowledgements. We would like to thank the referees of this paper for constructive comments and remarks. The $D_{\text {st }}$ data are provided by the World Data Center for Geomagnetism, Kyoto (http://swdcwww.kugi.kyoto-u.ac.jp/). The X-rays flux data are downloaded from the Space Physics Interactive Data Resource of the National Geophysical Data Center (http://spidr.ngdc.noaa.gov/spidr/).

Edited by: H. Lamy

Reviewed by: Z. Voros and another anonymous referee

\section{References}

Abe, S. and Bagci, G. B.: Necessity of q-expectation value in nonextensive statistical mechanics, Phys. Rev. E, 71, 016139/15, doi:10.1103/PhysRevE.71.016139, 2005.

Akay, M.: Time Frequency andWavelets in Biomedical Signal Processing Engineering, Wiley-IEEE Press, 768 pp., 1997.

Baiesi, M., Paczuski, M., and Stella, A. L.: Intensity Thresholds and the Statistics of the Temporal Occurrence of Solar Flares, Phys. Rev. Lett., 96, 051103/1-4, doi:10.1103/PhysRevLett.96.051103, 2006.

Balasis, G., Daglis, I. A., Kapiris, P., Mandea, M., Vassiliadis, D., and Eftaxias, K.: From pre-storm activity to magnetic storms: a transition described in terms of fractal dynamics, Ann. Geophys., 24, 3557-3567, doi:10.5194/angeo-24-3557-2006, 2006.

Balasis, G., Daglis, I. A., Papadimitriou, C., Kalimeri, M., Anastasiadis, A., and Eftaxias, K.: Dynamical complexity in Dst time series using non-extensive Tsallis entropy, Geophys. Res. Lett., 35, L14102, doi:10.1029/2008GL034743, 2008.

Balasis, G. and Eftaxias, K.: A study of non-extensivity in the Earth's magnetosphere, Eur. Phys. J.-Spec. Top., 174, 219-225, 2009

Balasis, G., Daglis, I. A., Papadimitriou, C., Kalimeri, M., Anastasiadis, A., and Eftaxias, K.: Investigating dynamical complexity in the magnetosphere using various entropy measures, J. Geophys. Res., 114, A00D06, doi:10.1029/2008JA014035, 2009.

Balasis, G., Daglis, I. A., Anastasiadis, A., Papadimitriou, C., Mandea, M., and Eftaxias, K.: Universality in solar flare, magnetic storm and earthquake dynamics using Tsallis statistical mechanics, Physica A, 390, 341-346, doi:10.1016/j.physa.2010.09.029, 2011a.

Balasis, G., Papadimitriou, C., Daglis, I. A., Anastasiadis, A., Athanasopoulou, L., and Eftaxias, K.: Signatures of discrete scale invariance in Dst time series, Geophys. Res. Lett., 38, L13103/1-6, doi:10.1029/2011GL048019, 2011 b.

Chang, T., Tam, S. W. Y., Wu, C. C., and Consolini, G.: Complexity, forced and/or self-organized criticality, and topological phase transitions in space plasmas, Space Sci. Rev., 107, 425445, 2003.

Chang, T., Tam, S. W. Y., and Wu, C. C.: Complexity in Space Plasmas - A Brief Review, Space Sci. Rev., 122, 281-291, doi:10.1007/s11214-006-5957-4, 2006.

Chang, T., Wu, C. C., Podesta, J., Echim, M., Lamy, H., and Tam, S. W. Y.: ROMA (Rank-Ordered Multifractal Analyses) of intermittency in space plasmas - a brief tutorial review, Nonlin. Processes Geophys., 17, 545-551, doi:10.5194/npg-17-545-2010, 2010.
Consolini, G., Chang, T., and Lui, A. T. Y.: Complexity and Topological Disorder in the Earth's Magnetotail Dynamics, Nonequilibrium Phenomena in Plasmas, edited by: Sharma, A. S. and Kaw, P. K., Springer, New York, 51-70, 2005.

Contoyiannis, Y., Kapiris, P., and Eftaxias, K.: Monitoring of a preseismic phase from its electromagnetic precursors, Phys. Rev. E, 71, 066123/1-14, doi:10.1103/PhysRevE.71.066123, 2005.

Daglis, I. A.: Ring current dynamics, Space Sci. Rev., 124, 183202, doi:10.1007/s11214-006-9104-z, 2006.

de Arcangelis, L., Godano, C., Lippiello, E., and Nicodemi, M.: Universality in Solar Flare and Earthquake Occurrence, Phys. Rev. Lett., 96, 051102/1-4, doi:10.1103/PhysRevLett.96.051102, 2006.

Eftaxias, K., Kapiris, P., Polygiannakis, J., Bogris, N., Kopanas, J., Antonopoulos, G., Peratzakis, A., and Hadjicontis, V.: Signatures of pending earthquake from electromagnetic anomalies, Geophys. Res. Lett., 28, 3321-3324, 2001.

Eftaxias, K., Frangos, P., Kapiris, P., Polygiannakis, J., Kopanas, J., Peratzakis, A., Skountzos, P., and Jaggard, D.: Review and a Model of Pre-Seismic electromagnetic emissions in terms of fractal electrodynamics, Fractals, 12, 243-273, 2004.

Eftaxias, K., Panin, V., and Deryugin, Y.: Evolution EM-signals before earthquake and during laboratory test of rocks, Tectonophysics, 431, 273-300, 2007.

Eftaxias, K., Athanasopoulou, L., Balasis, G., Kalimeri, M., Nikolopoulos, S., Contoyiannis, Y., Kopanas, J., Antonopoulos, G., and Nomicos, C.: Unfolding the procedure of characterizing recorded ultra low frequency, $\mathrm{kHZ}$ and $\mathrm{MHz}$ electromagetic anomalies prior to the L'Aquila earthquake as preseismic ones - Part 1, Nat. Hazards Earth Syst. Sci., 9, 1953 1971, doi:10.5194/nhess-9-1953-2009, 2009.

Eftaxias, K., Balasis, G., Contoyiannis, Y., Papadimitriou, C., Kalimeri, M., Athanasopoulou, L., Nikolopoulos, S., Kopanas, J., Antonopoulos, G., and Nomicos, C.: Unfolding the procedure of characterizing recorded ultra low frequency, $\mathrm{kHZ}$ and $\mathrm{MHz}$ electromagnetic anomalies prior to the L'Aquila earthquake as pre-seismic ones - Part 2, Nat. Hazards Earth Syst. Sci., 10, 275 294, doi:10.5194/nhess-10-275-2010, 2010.

Guttenberg, B. and Richter, C. F.: Frequency of earthquakes in California, Bull. Seismol. Soc. Am., 34, 185-188, 1944.

Hao, B.-L.: Elementary symbolic dynamics and chaos in dissipative systems, World Scientific, Singapore, 1989.

Isliker, H., Anastasiadis, A., and Vlahos, L.: MHD consistent cellular automata (CA) models II: Applications to solar flares, Astron. Astrophys., 377, 1068-1080, 2001.

Kalimeri, M., Papadimitriou, C., Balasis, G., and Eftaxias, K.: Dynamical complexity detection in pre-seismic emissions using nonadditive Tsallis entropy, Physica A, 387, 1161-1172, 2008.

Kapiris, P. G., Balasis, G. T., Kopanas, J. A., Antonopoulos, G. N., Peratzakis, A. S., and Eftaxias, K. A.: Scaling similarities of multiple fracturing of solid materials, Nonlin. Processes Geophys., 11, 137-151, doi:10.5194/npg-11-137-2004, 2004a.

Kapiris, P., Eftaxias, K., and Chelidze, T.: The electromagnetic signature of prefracture criticality in heterogeneous media, Phys. Rev. Lett., 92, 065702/1-4, doi:10.1103/PhysRevLett.92.065702, 2004b.

Kapiris, P., Polygiannakis, J., Li, X., Yao, X., and Eftaxias, K.: Similarities in precursory features in seismic shocks and epileptic seizures, Europhys. Lett., 69, 657-663, 2005. 
Karamanos, K., Peratzakis, A., Kapiris, P., Nikolopoulos, S., Kopanas, J., and Eftaxias, K.: Extracting preseismic electromagnetic signatures in terms of symbolic dynamics, Nonlin. Processes Geophys., 12, 835-848, doi:10.5194/npg-12-835-2005, 2005.

Karamanos, K., Dakopoulos, D., Aloupis, K., Peratzakis, A., Athanasopoulou, L., Nikolopoulos, S., Kapiris, P., and Eftaxias, K.: Study of pre-seismic electromagnetic signals in terms of complexity, Phys. Rev. E, 74, 016104/1-21, doi:10.1103/PhysRevE.74.016104, 2006.

Kossobokov, V., Keillis-Borok, V., and Cheng, B.: Similarities of multiple fracturing on a neutron star and on Earth, Phys. Rev. E, 61, 3529-3533, 2000.

Lavenda, B. H. and Dunning-Davies, J.: Additive Entropies of degree-q and the Tsallis Entropy, arXiv:physics/0310117, 2003a.

Lavenda, B. H. and Dunning-Davies, J.: Qualms concerning Tsalliss condition of Pseudo-Additivity as a Definition of NonExtensivity, arXiv:cond-mat/0311477, 2003b.

Leubner, M. P. and Vörös, Z.: A nonextensive entropy path to probability distributions in solar wind turbulence, Nonlin. Processes Geophys., 12, 171-180, doi:10.5194/npg-12-171-2005, 2005.

Matcharashvili, T., Chelidze, T., Javakhishvili, Z., Jorjiashvili, N., and Fra Paleo, U.: Non-extensive statistical analysis of seismicity in the area of Javakhety, Georgia, Comput. Geosci., doi:10.1016/j.cageo.2010.12.008, in press, 2011.

Nauenberg, M.: Critique of q-entropy for thermal statistics, Phys. Rev. E, 67, 036114, doi:10.1103/PhysRevE.67.036114, 2003.

Papadimitriou, C., Kalimeri, M., and Eftaxias, K.: Nonextensivity and universality in the earthquake preparation process, Phys. Rev. E., 77, 036101/1-14, doi:10.1103/PhysRevE.77.036101, 2008

Pulinets, S. A. and Boyarchuk, K. A.: Ionospheric Precursors of Earthquakes, Springer, Berlin, Germany, 315 pp., 2004.

Silva, R., Franca, G., Vilar, C., and Alcaniz, J.: Nonextensive models for earthquakes, Phys. Rev. E, 73, 026102/1-5, doi:10.1103/PhysRevE.73.026102, 2006.

Sornette, D.: Critical Phenomena in Natural Sciences, Springer, Heidelberg, 528 pp., 2004.
Sornette, D. and Helmstetter, A.: Occurrence of finitetime singularities in epidemic models of rupture, earthquake, and starquakes, Phys. Rev. Lett., 89, 158501/1-4, doi:10.1103/PhysRevLett.89.158501, 2002.

Sotolongo-Costa, O. and Posadas, A.: Fragment-asperity interaction model for earthquakes, Phys. Rev. Lett., 92, 048501/1-4, doi:10.1103/PhysRevLett.92.048501, 2004.

Telesca, L.: Nonextensive analysis of seismic sequences, Physica A, 389, 1911-1914, 2010.

Telesca, L. and Chen, C.-C.: Nonextensive analysis of crustal seismicity in Taiwan, Nat. Hazards Earth Syst. Sci., 10, 1293-1297, doi:10.5194/nhess-10-1293-2010, 2010.

Tsallis, C.: Possible generalization of Boltzmann-Gibbs statistics, J. Stat. Phys., 52, 479-487, 1988.

Tsallis, C.: Introduction to Nonextensive Statistical Mechanics, Approaching a Complex Word, Springer, New York, 382 pp., 2009.

Turcotte, D. L.: Fractals and Chaos in Geology and Geophysics, Cambridge Univ. Press, 398 pp., 1997.

Vassiliadis, D., Anastasiadis, A., Georgoulis, M., and Vlahos, L.: Derivation of solar flare cellular automata models from a subset of the magnetohydrodynamic equations, Astroph. J. Lett., 509, L53-L56, 1998.

Wanliss, J. A.: Fractal properties of SYM-H during quiet and active times, J. Geophys. Res., 110, A03202, doi:10.1029/2004JA010544, 2005.

Wanliss, J. A., Anh, V. V., Yu, Z.-G., and Watson, S. M.: Multifractal modeling of magnetic storms via symbolic dynamics analysis, J. Geophys. Res., 110, A08214, doi:10.1029/2004JA010996, 2005.

Zanette, D. H. and Montemurro, M. A.: A note on nonthermodynamical applications of non-extensive statistics, Phys Lett. A, 324, 383-387, 2004.

Zhou, C., Motter, A. E., and Kurths, J.: Universality in the synchronization of weighted random networks, Phys. Rev. Lett., 96 , 034101/1-4, doi:10.1103/PhysRevLett.96.034101, 2006. 